\title{
Numerical Feasibility Study of Electric Heating Strategies for Subsea Tie-In Flowlines Using a 1-D Mechanistic Multiphase Flow Simulator
}

\author{
Diana González, Milan Stanko, Michael Golan \\ Department of Geoscience and Petroleum, The Norwegian University of Science and Technology (NTNU), Trondheim, Norway \\ Email: diana.rengifo@ntnu.no
}

How to cite this paper: González, D., Stanko, M. and Golan, M. (2018) Numerical Feasibility Study of Electric Heating Strategies for Subsea Tie-In Flowlines Using a 1-D Mechanistic Multiphase Flow Simulator. Engineering, 10, 561-571. https://doi.org/10.4236/eng.2018.109040

Received: July 16, 2018

Accepted: August 28, 2018

Published: August 31, 2018

Copyright (c) 2018 by authors and Scientific Research Publishing Inc. This work is licensed under the Creative Commons Attribution International License (CC BY 4.0).

http://creativecommons.org/licenses/by/4.0/

\begin{abstract}
This paper reports the outcome of a study investigating four electrical heat-tracing strategies that can be employed to avoid wax deposition in subsea flowlines transporting untreated crude oil from subsea wells to a host installation. The strategies, described in the paper, are distinct by the configuration of deploying the heat tracing along the pipe and by the activation schedule (continuous or alternating heating cycles). The study demonstrates quantitatively that the electrical power required for maintaining wax-free flow depends strongly on the employed strategy. The complex thermo-hydraulic flow was modeled using a commercial Dynamic Multiphase Flow Simulator with wax deposition option and thermal insulation as an input parameter. In conclusion, the paper ranks the strategies according to their computed energy efficiency, thus providing quantitative benchmarks for preliminary wax control assessment.
\end{abstract}

\section{Keywords}

Flow Assurance, Wax Deposition, Electric Heating, Pipeline Insulation

\section{Introduction}

The subsea production and transport of untreated reservoir fluids require overcoming phenomena that restrict the flow or disturb its smoothness. Challenging these problems is referred to, in the petroleum industry, as Flow Assurance. This paper addresses one particular problem, Wax deposition, occurring often in flow of untreated reservoir oil in wells, surface and subsea production systems, and flowlines conducted untreated oil.

Wax is a compound consisted of heavy paraffinic components which are fully 
dissolved in the hydrocarbon mixture at high temperature, but tends to precipitate as a solid-like soft material when the temperature declines below a certain level referred to as Wax Appearance Temperature (WAT). Upon temperature decline of the homogenous liquid phase, the WAT marks the inception of wax appearance and solid participation from the liquid phase. The WAT is also referred to as Cloud Point due to the appearance of visible cloud-like compound of small-dispersed wax crystals. The point depends on the molecular composition of the particular hydrocarbon fluid and is determined and reported for the various crude oil systems according to well-established industry standards and test procedures.

The formed wax tends to deposit on the pipe wall, as it is normally colder than the core of the flowing stream, forming a layer of sticky solid paste that restricts the flow. The consequence is progressive reduction of flow rate, or increase in flowing pressure, which might end up in near complete or even complete clogging of the pipe.

Calculation models to predict the wax build-up in a pipe cross section are based on molecular diffusion mechanism of wax crystals driven by concentration gradient from the center outwards. This is combined with shear dispersion of the accumulated wax crystals by the flowing fluid mechanisms. Such models are used today in engineering calculations for predicting wax build-up and for mapping wax restriction along pipe sections with constant or declining flow stream temperature. Computational wax build-up routine can be linked to flow simulator and they are often built-in in complex flow simulators.

The industry developed several practices to mitigate wax deposition based on three approaches: 1) chemical, 2) thermal, and, 3) mechanical. Each approach has upsides and downsides with respect to investment, operations, reliability, and environmental impact.

The chemical method consists of injecting inhibitors into the pipeline stream [1]. These include crystal-modifier-type inhibitors, dispersants, and solvents. The mechanical approach is based on a flow stream driving (hydrostatically) of a piston-like "pig" which cuts, disperses, and pushes the wax in the flow direction towards the pipe exit end. The pigging process requires a pig launcher arrangement at the inlet to the pipe and a pig receiver and cuttings recovery arrangement at the pipe outlet. The complication of pigging a subsea production system stems from the need to locate both the launcher and the receiver on the host installation, which implies a costly pigging loop. Alternatively, a subsea pig launcher eliminates the pigging loop but this solution is prohibited when frequent pigging is needed (sometimes on weekly bases or more).

The thermal approach intended to keep the pipe flowing streams above the Wax Appearance Temperature.

There are two types of thermal management practices: passive (pipe thermal insulation) and active (heating). Thermal insulation is not effective for long transportation distances. In such cases, the stream temperature drops continuously along the pipeline and will inevitably reach the wax appearance temper- 
ature. The active heat management, also known as "Active Heating" is based on heating the line to avoid wax deposition. Interestingly, active heating of oil line to inhibit wax forming is analog to active heating to inhibit hydrates forming in untreated natural gas lines.

Active heating provides a cost-effective alternative for the managing of hydrates and wax in production pipelines since the early 1990's. The existing active heating technologies are categorized as: 1) Pipe-in-Pipe Technology (PiP) based on a concentric hot fluid flow envelop around the hydrocarbon line, and 2) electrical heating, or electrical heat tracing. Heat tracing is the subject of this paper.

Many studies, which evaluate the efficiency of electric heating technologies, can be found in the literature. Denniel and Laouir [2], worked on the development of heated PiP technology and demonstrated its high efficiency, where low linear power inputs (20 to $40 \mathrm{~W} / \mathrm{m}$ of pipe) were sufficient for the prevention of hydrate or wax formation. Later, they applied the technology to a $20 \mathrm{~km}$ tie-back to maintain indefinitely the fluid temperature $26^{\circ} \mathrm{C}$ above ambient temperature. Escobar-Remolina, Barrios and Silva [3] applied electric heating technology to a well production line along 900 meter in a Colombian field, where an extra-heavy oil was been produced. They managed to increase the daily oil production of the well in 160\%. Pedersen, Kullbotte, Børnes and Marthinsen [4], performed laboratory experiments where an ice plug was formed in a $4.5 \mathrm{~m}$ long 30 pipe joint and melted using DEH. The experiments showed that $3 \%$ of the ice plug got melted at an ambient temperature of $-2{ }^{\circ} \mathrm{C}$ and a current of $1300 \mathrm{~A}$ for 48 hours, while increasing the current to $1500 \mathrm{~A}$ melted $6 \%$ of the ice plug in the same period of time. Decrin, Nebell, De Naurois and Parenteau [5] discussed the modeling $\mathrm{DEH}-\mathrm{PiP}$ to predict the production fluid behavior during transient active heating operations using CFD and multiphase flow simulator (OLGA or Leda). They validated the models in the frame of TOTAL's Islay development during the shut-in period. Tzotzi et al. [6] presented the hydrate formation inside an EHT-PiP system and its safe and controlled dissociation using active heating. Finally, Lirola, Pouplin, Settouti and Agoumi [7] presented an overview of two active heating solutions: distributed and local; as a conclusion, they provided guidelines about which technology is the most adequate depending on the oil properties.

This paper presents a numerical feasibility study of electric heating strategies to avoid the formation of wax particles in subsea flowlines. The commercial software simulator OLGA 7.2.1 and a synthetic production system that represents a typical oil field in the North Sea were used. Four strategies were analyzed on two pipeline lengths, $8 \mathrm{~km}$, and $50 \mathrm{~km}$. The first strategy consists of continuous heating of the whole pipeline during a period of 90 days. In the second strategy, only a section of the pipeline was continuously heated, the beginning of this section is the point, in the pipeline, where the bulk fluid temperature reaches the WAT. The third strategy consists of intermittent heating of the whole flowline. In the last strategy, only applied in the $8 \mathrm{~km}$ pipeline, the flow line was continuously heating in sections (localized heating) of $100 \mathrm{~m}$ and 500 
$\mathrm{m}$. The influence of the pipeline insulation was also studied by performing the analysis with and without insulation. All strategies were compared using their average power consumption.

\section{Wax Deposition}

\subsection{Wax Deposition Mechanisms}

There are several models available in the literature to study the wax deposition mechanisms [8]; however, the two most widely used are molecular diffusion and shear dispersion.

\subsubsection{Molecular Diffusion}

Molecular diffusion is the process where the wax particles, dissolved in the bulk flow, are diffused toward the pipe wall, where they precipitate, creating then a concentration gradient. Based on the results of Bern, Withers and Cairns [9] and Brown, Niessen and Erickson [10], molecular diffusion is the principal responsible for wax deposition, this is especially valid for systems at high temperatures and high heat flux conditions [11].

\subsubsection{Shear Dispersion}

Shear dispersion is a phenomenon which causes the transport of the precipitated wax to be predominantly far from the wall. Since the suspended wax particles move in the same direction and speed of the flow, they tend to travel to the center of the pipe due to the flow velocity gradient. Brown, Niessen and Erickson [10] indicate that the effect of shear dispersion is not significant in comparison with molecular diffusion. On the other hand, Bern, Withers and Cairns [9] and Burger, Perkins and Striegler [11], stated that shear dispersion is relevant when the flow is at low temperature and low heat flux, and when there is a high concentration of wax crystals in the flow.

\subsection{Wax Deposition Modelling in the Commercial Transient Multiphase Simulator-OLGA 7.2.1}

OLGA 7.2.1simulator [12] has the wax deposition module to model wax precipitation and deposition. The calculation is made based on a pre-calculated table that contains information on each wax-forming component. This simulator has three models for wax deposition, they are RRR (Rygg, Rygdahl, and Rønningsen) model, Matzain model and HEATANALOGY model.

\subsubsection{RRR (Rygg, Rydahl and Rønningsen) Model}

The RRR model was created by Rygg, Rydahl, and Rønningsen in 1998 [8] and it predicts wax deposition in multiphase flow in pipelines. The model takes into account both molecular diffusion and shear dispersion effects, and the effect of wax deposition in the pressure drop due to the decreases of the diameter. In addition, the model includes the insulation effect of the wax layer that influences the heat exchange between the fluids and the environment. 


\subsubsection{Matzain Model}

The Matzain is a semi-empirical kinetic model that predicts wax thickness. The model takes into account shear stripping together with molecular diffusion and shear dispersion as wax deposition mechanisms, being shear stripping a wax reducing mechanism [8].

\subsubsection{Heat Analogy}

This is a proprietary model for wax deposition in pipelines used by OLGA 7.2.1. The model takes into account molecular diffusion, shear dispersion and shear stripping as mechanisms of deposition. No further details of the model are provided in the manual of the software.

\section{Methodology}

Four heating strategies to avoid the formation of wax particles in subsea pipeline, were applied to a synthetic field. The strategies consist of warming up the wall of the pipeline to keep the bulk flow temperature above the wax appearance temperature. The heating effect results from the heat generated when an electric current flows through a conductive material. The four strategies are:

- Strategy 1: Continuous heating of the whole pipeline. For a period of 90 days, the total length of the pipeline was continuously heated and the minimal power required to keep the bulk flow above the WAT was calculated.

- Strategy 2: Continuous heating of a section of the pipeline. Here, the pipeline was divided into two sections. In the first section, which goes from the beginning of the flowline until a certain length, the flow was allowed to naturally cool down. In the other section, which goes from the end of the first section to the end of the pipeline, the wall of the pipeline was continuously heated to maintain the flow over the WAT. The point, in the pipeline, at which the temperature of the flow reaches the WAT, determines the length of the sections. For the pipeline of $8 \mathrm{~km}$, the first $2.4 \mathrm{~km}$ were not heated and the heat was applied in the remaining $5.6 \mathrm{~km}$. For the pipeline of $50 \mathrm{~km}$, the heating was applied in a section of $49.7 \mathrm{~km}$. The minimal power required to avoid wax formation in the pipeline inner wall was calculated.

- Strategy 3: Intermittent heating of the whole pipeline. The total length of the pipeline was intermittently heated by periods of 1 day, 12 hours, 8 hours and 4 hours respectively, over 90 days. The minimal power consumption required to avoid wax formation was calculated.

- Strategy 4: Continuous heating of the pipeline by sections. In this strategy, the pipeline was divided into sections of equal lengths and heat was applied every other section for a period of 90 days. Two section lengths were tested: $500 \mathrm{~m}$ and $100 \mathrm{~m}$. The minimal power required was calculated.

The strategies were tested with two pipeline lengths: $8 \mathrm{~km}$ and $50 \mathrm{~km}$. The four strategies were applied in the $8 \mathrm{~km}$ pipeline, while only strategies 1,2 and 3 were used in the $50 \mathrm{~km}$ pipeline. The forth strategy was not implemented in the $50 \mathrm{~km}$ pipeline due to the high amount of sections, which made difficult the se- 
tup of the simulation.

The influence of the insulation was also studied. For this, all the strategies were tested twice, with insulation and without insulation in the pipeline.

\section{Study Case}

The production system used consists of a horizontal pipeline, with an internal diameter of $17 \mathrm{~cm}$. The fluid enters the pipeline with a mass flow of $17.51 \mathrm{~kg} / \mathrm{s}$, which represents a standard flow rate of $14 \mathrm{e} 03 \mathrm{Sm} 3 / \mathrm{d}$ for 32 API HC gravity, the gas specific gravity of 0.95 , an oil specific gravity of 0.87 , a temperature of $70^{\circ} \mathrm{C}$, a zero-water cut, a GVF of 0.085 and a WAT of $22^{\circ} \mathrm{C}$. The outlet pressure was 25 bara. The surrounding temperature was $5^{\circ} \mathrm{C}$. The external coefficient of convective heat transfer with the seawater is $500 \mathrm{~W} / \mathrm{m}-\mathrm{K}$. Figure 1 shows a sketch of the flowline diagram used in OLGA.

The pipeline wall material was steel, while the insulation was composed of concrete and polypropylene. Table 1 shows the properties of these materials.

The wax deposition model used was RRR. The total simulation time was 90 days, with a maximum time step of $1000 \mathrm{~s}$ and a minimum of $1 \mathrm{~s}$. The information given above was used for all the strategies.

Two criteria were used to determine the minimum required power. In one criterion, the fluid temperature in the pipeline cannot be less than the WAT. The second criterion, defined by the authors and applied in strategies 3 and 4 , is that the maximum thickness of the wax layer deposited in the pipeline cannot be higher than $1 \%$ of the pipeline ID.

\section{Results and Discussion}

The aim of the results is to generate conclusions about the efficiency of each active heating strategy studied and to rank them based on this criterion. The authors chose to quantify the efficiency of a strategy by computing its average power

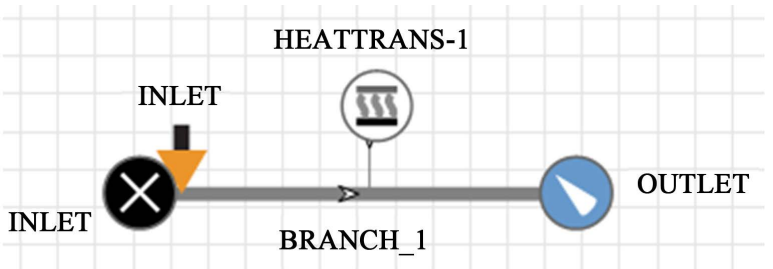

Figure 1. Schematic flowline diagram used in OLGA.

Table 1. Wall material's properties.

\begin{tabular}{cccc}
\hline & Steel & Concrete & Polypropylene \\
\hline Thickness $[\mathrm{cm}]$ & 1.00 & 0.60 & 0.30 \\
Conductivity $[\mathrm{W} / \mathrm{m}-\mathrm{K}]$ & 50.00 & 1.70 & 0.12 \\
Density $\left[\mathrm{kg} / \mathrm{m}^{3}\right]$ & 7850 & 2250 & 960 \\
Capacity $[\mathrm{J} / \mathrm{kg}-\mathrm{K}]$ & 485 & 880 & 1675 \\
\hline
\end{tabular}


consumption per meters of pipeline. The lower the average power consumption is, the more efficient the strategy is.

It is important to highlight that the results showed for the third strategy (intermittent heating of the whole pipeline) correspond to the heating period of 1 day, as the most efficient period with the lower percentage of wax formation along the pipeline.

Figure 2 shows the required power consumption per meter for both pipeline lengths with insulation. For this case, strategy 1 and strategy 2 were the less power-consuming strategies, both for the $8 \mathrm{~km}$ and the $50 \mathrm{~km}$ pipelines, with a power consumption per meter no greater than $400 \mathrm{~W} / \mathrm{m}$. On the other hand, for both pipeline lengths, strategy 3 tripled the energy requirements with a power consumption per meter of $1500 \mathrm{~W} / \mathrm{m}$, been the less energy-efficient strategy. Strategy 4, only applied to the $8 \mathrm{~km}$ pipeline, had an intermediate power consumption per meter of $850 \mathrm{~W} / \mathrm{m}$ for sections of $500 \mathrm{~m}$ and $750 \mathrm{~W} / \mathrm{m}$ for sections of $100 \mathrm{~m}$.

Figure 3 shows the required power consumption for both pipeline lengths when there is no insulation in the pipelines. In this case, the power consumption for all studied strategies increased considerably in comparison with the case when the pipelines have insulation. Strategies 1 and 2 were still the most power-efficient. However the required power consumption per meter increased to

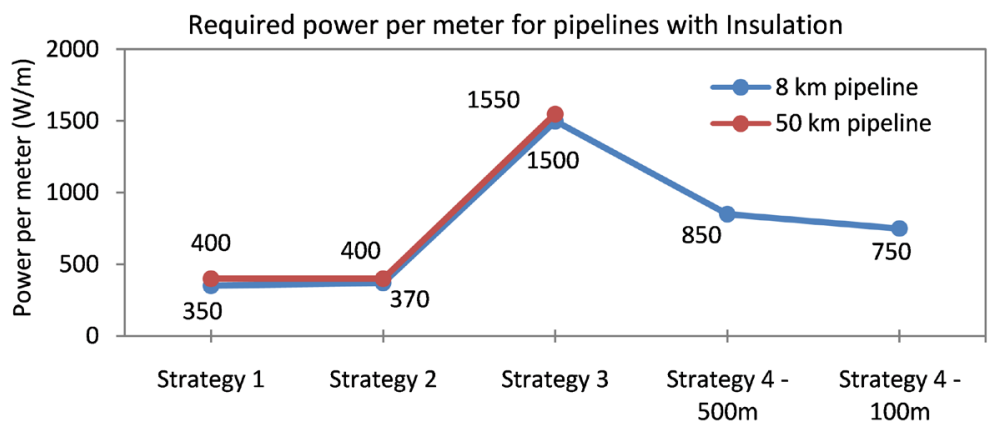

Figure 2. Required power consumption per meter of pipeline with insulation for both pipeline lengths, $8 \mathrm{~km}$ and $50 \mathrm{~km}$.

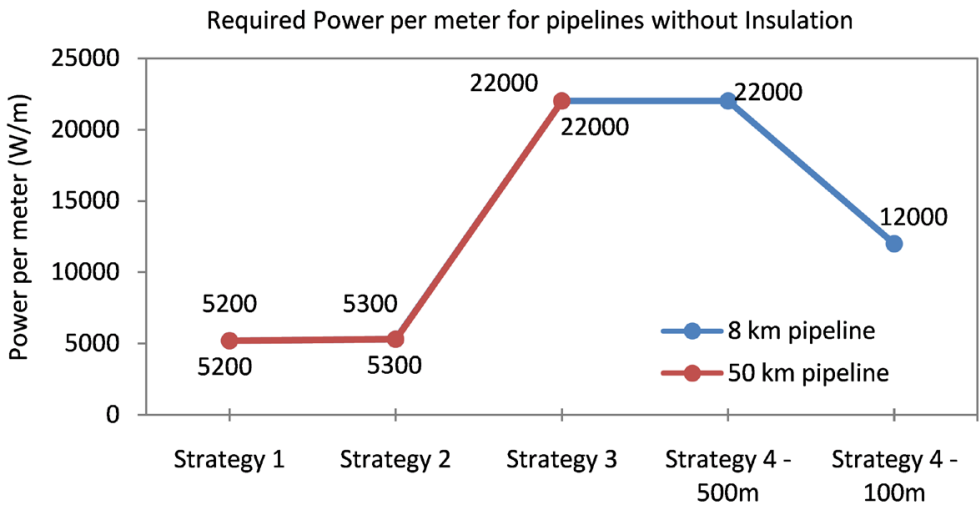

Figure 3. Required power consumption per meter of pipeline without insulation for both pipeline lengths, $8 \mathrm{~km}$ and $50 \mathrm{~km}$. 
$5200 \mathrm{~W} / \mathrm{m}$ in strategy 1 and $5300 \mathrm{~W} / \mathrm{m}$ in strategy 2, for both pipeline lengths. For strategy 3 (both pipeline lengths) and strategy 4, with pipeline segments of $500 \mathrm{~m}$, the power consumption per meter was $22,000 \mathrm{~W} / \mathrm{m}$, resulting in the less power-efficient strategies. Strategy 4 with pipeline segments of $100 \mathrm{~m}$, had intermediate power consumption of $12,000 \mathrm{~W} / \mathrm{m}$.

Based on the results obtained, one can conclude that to avoid wax formation, it is best to continuously heat the entire pipeline or its critical section, where the fluid temperature drops below the WAT. This means that strategies 1 and 2 are the most efficient compared to the others, been their power consumption per meter less than half of that required by the other strategies.

The less efficient strategy is intermittent heating. This strategy has non-heating periods where, for all the cases analyzed ( $1 \mathrm{~d}, 12 \mathrm{hr}, 8 \mathrm{hr}$ and $4 \mathrm{hr}$ ), the fluid temperature drops below the WAT (where wax can potentially precipitate). High power rates are then required to heat the fluid again to a temperature above the WAT.

In strategy 4 , where the heating was applied in intercalated segments of equal lengths, the power required in the heated sections depends on the segment length. The power has to be such that, it guarantees that the fluid temperature will not drop below the WAT in the non-heated sections. In other words, the temperature at the end of the heated sections has to be high enough to avoid wax formation in the subsequent non-heated sections.

Pipeline insulation helps to reduce the heat transfer between the bulk flow and the ambient. Thus, the power needed to heat the fluid above a certain temperature is lower when the pipeline has insulation. It was observed that, on average, a decrease of $86 \%$ in the overall heat transfer coefficient leads to a decrease of $93 \%$ in the required power.

To confirm the results obtained, the total power consumption is analyzed below. Figure 4 and Figure 5 show the total power consumption of all studied strategies for both pipeline lengths, and for pipelines with insulation (Figure 4) and without insulation (Figure 5). In both figures, it can be seen that strategies 1 and 2 were still the most power-efficient strategies, while the less efficient was strategy 3 .

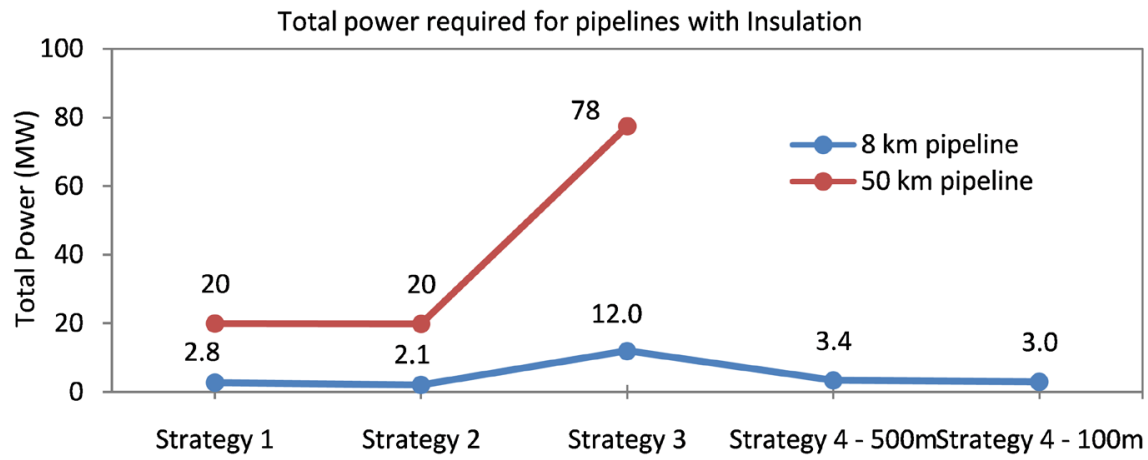

Figure 4. Total power required for pipelines with insulation. 


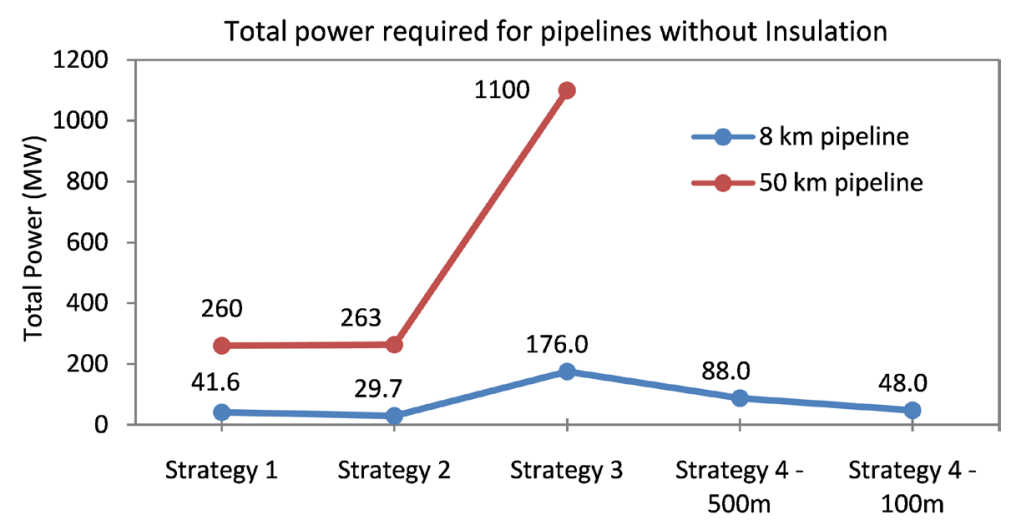

Figure 5. Total power required for pipeline without insulation.

In strategy 1 , the power consumption per meter, along the $8 \mathrm{~km}$ pipeline, was at most $5 \%$ lower than the one in strategy 2 . In other words, strategy 2 required a higher power capacity installed in the pipeline. On the other hand, the total power consumption of strategy 1 was $33 \%$ higher than strategy 2 , when the pipeline has insulation (Figure 4), and 40\% higher than strategy 2 for pipeline without insulation (Figure 5). This means that, for this specific case, where the pipeline length was $8 \mathrm{~km}$ and the heated section in strategy 2 was $5.6 \mathrm{~km}$, it is most energy-efficient to apply strategy 2.

In the case of the $50 \mathrm{~km}$ pipeline, for pipeline with insulation, the total power consumption of strategy 1 and 2 was the same. For pipeline without insulation, the total power consumption of strategy 1 was $1.15 \%$ lower than for strategy 2 . Since the heated section in strategy 2 had almost the same length as in strategy 1 , the total power consumption was similar for both strategies.

Figure 4 and Figure 5 confirms that strategies 3 and 4 require the highest power consumption, been the less power-efficient one, strategy 3 , intermittent heating.

It is important to highlight that the total power consumption depends on the pipeline length. It is economically more efficient to apply heating to the shorter pipelines since they require less power consumption.

\section{Conclusions}

Four strategies of electric heating were studied for two pipeline lengths, $8 \mathrm{~km}$, and $50 \mathrm{~km}$. Their required power consumption was compared with each other and the most efficient strategies were determined. At the same time, the effect of having insulation in the pipeline was addressed.

- The most power-efficient strategies are strategy 1 and strategy 2, where the pipeline or a section of it, is continuously heated.

- The less power-efficient strategy is intermittent heating of the pipeline (strategy 3), since the fluid needs to be heated above the WAT after every non-heated period.

- For strategy 4, heating by sections, it is important to determine an optimal section length that guarantees the lower power consumption and the lower 
formation of wax in the no-heated sections.

- We have also concluded that insulation plays an important role in active heating, since it reduces the heat transfer between the fluid and its surroundings, lowering the power requirements. A decrease of $86 \%$ in the overall heat transfer coefficient led to a decrease of $93 \%$ in the required power.

- Finally, the total power consumption of any strategy depends on the length of the pipeline to be heated.

\section{Conflicts of Interest}

The authors declare no conflicts of interest regarding the publication of this paper.

\section{References}

[1] Kondapi, P. and Moe, R. (2013) Today's Top 30 Flow Assurance Technologies: Where Do They Stand? Offshore Technology Conference, Houston, 6-9 May 2003, OTC 24250

[2] Denniel, S. and Laouir, N. (2001) Active Heating for Ultra-Deepwater PiP and Risers. Offshore Technology Conference, Houston, 31 April-3 May 2001, OTC 13138. https://doi.org/10.4043/13138-MS

[3] Escobar-Remolina, J., Barrios, W. and Silva, B. (2012) Production Increase with Electric Heating Production Line Technology in an Extra-Heavy Oil Field in Colombia: Successful Case of Flow Assurance. SPE Annual Technical Conference and Exhibition, San Antonio, 8-10 October 2012, SPE 159219.

[4] Pedersen, A., Kullboteen, H., Børnes, A. and Marthinsen, T. (2007) Laboratory Experiments of Melting Ice Plugs in Pipelines by Applying Direct Electric Heating. Seventeenth International Offshore and Polar Engineering Conference, Lisbon, 1-6 July 2007.

[5] Decrin, M.-K., Nebell, F., De Naurois, H. and Parenteau, T. (2013) Flow Assurance Modeling Using an Electrical Trace Heated Pipe-in-Pipe: From Qualification to Offshore Testing. Offshore Technology Conference, Houston, 6-9 May 2013, OTC 24060 .

[6] Tzotzi, C., Parenteau, T., Kaye, D., Turner, D., Bass, R., Morgan, J., Zakarian, E., Rolland, J. and Decrin, M.-K. (2016) Safe Hydrate Plug Dissociation in Active Heating Flowlines and Risers-Full Scale Test. Offshore Technology Conference, Houston, 2-5 May 2016, OTC-27051-MS.

[7] Lirola, F., Pouplin, A., Settouti, N. and Agoumi, J. (2017) Technical Assessment and Qualification of Local and Distributed Active Heating Technologies. 13th Offshore Mediterranean Conference and Exhibition, Ravenna, 29-31 March 2017.

[8] Rosvol, K. (2008) Wax Deposition Model. Master Thesis, NTNU, Trondheim.

[9] Bern, P.A., Withers, V.R. and Cairns, J.R. (1980) Wax Deposition in Crude Oil Pipelines. European Offshore Petroleum Conference and Exhibition, London, 21-24 October 1980, EUR 206. https://doi.org/10.2118/206-1980-MS

[10] Brown, T.S., Niessen, V.G. and Erickson, D. (1993) Measurement and Prediction of the Kinetics of Paraffin Deposition. $68^{\text {th }}$ Annual Technical Conference and Exhibition of the Society of Petroleum Engineers, Houston, 3-6 October 1993, SPE 26548. https://doi.org/10.2118/26548-MS 
[11] Burger, E.D., Perkins, T.K. and Striegler, J.H. (1981) Studies of Wax Deposition in the Trans Alaska Pipeline. Journal of Petroleum Technology, 33, 1075-1086. https://doi.org/10.2118/8788-PA

[12] OLGA 7.2.1. User Manual. 